\title{
Seasonal Variations in Stroke: A Study in a Hospital in North India
}

\author{
Pradeep Kumar, Amit Kumar, Awadh Kishor Pandit, Abhishek Pathak, Kameshwar Prasad \\ Department of Neurology, All India Institute of Medical Sciences, New Delhi, India
}

\section{Dear Sir:}

Stroke accounts for nearly 5.7 million deaths worldwide; $87 \%$ of these deaths occur in low- and middle-income countries. ${ }^{1,2}$ India is a tropical country with various seasons that may influence stroke occurrence. ${ }^{3}$ The mechanisms underlying these seasonal variations of stroke are not fully understood, but a possible reason may include seasonal variation of biological factors such as arterial blood pressure, serum lipid levels, and other blood components. ${ }^{4,5}$ This study aimed to determine if there is any evidence of seasonal variation in the occurrence of various subtypes of strokes in North India.

Data were collected from patients with a first-ever stroke who were admitted to the neurology ward of a tertiary care hospital in North India from January 2009 to April 2014. The diagnosis of ischemic stroke (IS) or intracerebral hemorrhage (ICH) was confirmed by neurologic examination and neuroimaging. We excluded secondary ICH, i.e., subarachnoid hemorrhage, arteriovenous malformation, and venous stroke. The collected data were patient name, age, sex, date, month, and year of stroke onset, type of stroke, hospital registration number, date of admission, and record of arterial blood pressure at the time of admission. Seasons were categorized into four categories: spring (March to May), summer (June to August), autumn (September to November), and winter (December to February). Statistical analysis was performed using Statistical Package for Social Science (SPSS) version 17 software. Frequency, mean and standard deviation (SD) values were determined for stroke type, age, and seasonal occurrence of stroke. The seasonal occurrence of stroke was evaluated with the chi-square test and Student's $t$ test, with the level of significance set at $P<0.05$.

Our analysis included 1,099 stroke patients, 769 of whom were male $(69.9 \%)$. The mean $( \pm S D)$ age was $53.8( \pm 15.7)$ years. A total of 663 patients $(60.3 \%)$ had IS and 436 patients (39.7\%) had ICH. IS was most prominent in spring $(n=223$, $33.6 \%)$, followed by the winter $(n=168,25.3 \%)$, summer $(n=$ $166,25 \%)$, and autumn $(\mathrm{n}=106,16 \%)$. The occurrence of ICH was most prominent in winter $(n=165,37.8 \%)$, followed by spring $(n=116,26.6 \%)$, autumn $(n=81,18.6 \%)$, and summer $(n=74,17 \%)$. The highest occurrence of IS was observed in April, whereas the occurrence of ICH was the highest in January. Significant seasonal differences in the occurrence of IS and ICH were observed in both men and women (Table 1). There were no seasonal variations in blood pressure between stroke types.

The highest incidence of IS in spring and ICH in winter agrees with previous reports from Japan, ${ }^{6,7}$ suggesting that the seasonal variation observed in northern Asia is also present in India. Our study, however, is limited in that it is from a single center, and detailed stroke subtypes (e.g., large artery disease, small artery disease, cardiogenic infarction) could not be assessed. Moreover, although seasonal variation of blood pressure was not observed in our study, this retrospective analysis did not allow us to assess biological factors as a potential cause of seasonal variation in stroke incidence. Further prospective studies with serial assessment of risk factors should be carried out to better understand the reasons for seasonal differences in the incidence of stroke. 
Table 1. Distribution of stroke types according to sex and season

\begin{tabular}{|c|c|c|c|c|c|c|c|c|}
\hline \multirow{2}{*}{ Sex } & & & \multicolumn{4}{|c|}{ Seasons } & \multirow{2}{*}{ Total [n] } & \multirow{2}{*}{$P$ value } \\
\hline & & & Spring [n (\%)] & Summer [n(\%)] & Autumn [n (\%)] & Winter [n (\%)] & & \\
\hline \multirow[t]{3}{*}{ Male } & Stroke & IS & 158 (33.8) & $115(24.6)$ & $78(16.7)$ & $117(25.0)$ & 468 & 0.001 \\
\hline & Type & $\mathrm{ICH}$ & $80(26.6)$ & 51 (16.9) & $60(19.9)$ & $110(36.5)$ & 301 & \\
\hline & Total & & 238 (30.9) & 166 (21.6) & $138(17.9)$ & 227 (29.5) & 769 & \\
\hline \multirow[t]{3}{*}{ Female } & Stroke & IS & 65 (33.0) & 51 (26.3) & $28(14.4)$ & 51 (26.3) & 195 & 0.026 \\
\hline & Type & $\mathrm{ICH}$ & $36(26.7)$ & $23(17)$ & 21 (15.6) & $55(40.7)$ & 135 & \\
\hline & Total & & 101 (30.4) & 74 (22.5) & $49(14.9)$ & 106 (32.2) & 330 & \\
\hline
\end{tabular}

IS, ischemic stroke; ICH, intracerebral hemorrhage.

\section{References}

1. Feigin VL, Forouzanfar MH, Krishnamurthi R, Mensah GA, Connor M, Bennett DA, et al. Global and regional burden of stroke during 1990-2010: findings from the Global Burden of Disease Study 2010. Lancet 2014;383:245-254.

2. Strong K, Mathers C, Bonita R. Preventing stroke: saving lives around the world. Lancet Neurol 2007;6:182-187.

3. Raj K, Bhatia R, Prasad K, Srivastava MV, Vishnebhatla S, Singh $\mathrm{MB}$. Seasonal differences and circadian variation in stroke occurrence and stroke subtypes. J Stroke Cerebrovasc Dis 2015; 24:10-16.

4. Rothwell PM, Wroe SJ, Slattery J, Warlow CP. Is stroke incidence related to season or temperature? The Oxfordshire Community Stroke Project. Lancet 1996;347:934-936.

5. Gordon DJ, Hyde J, Trost DC, Whaley FS, Hannan PJ, Jacobs $\mathrm{DR}$, et al. Cyclic seasonal variation in plasma lipid and lipoprotein levels: the lipid research clinics coronary primary prevention trial placebo group. J Clin Epidemiol 1988;41:679-689.
6. Turin TC, Kita Y, Murakami Y, Rumana N, Sugihara H, Morita $\mathrm{Y}$, et al. Higher stroke incidence in the spring season regardless of conventional risk factors. Takashima Stroke Registry, Japan, 1988-2001. Stroke 2008;39:745-752.

7. Takizawa S, Shibata T, Takagi S, Kobayashi S. Japan Standard Stroke Registry Study Group. Seasonal variation of stroke incidence in Japan for 35631 stroke patients in the Japanese Standard Stroke Registry, 1998-2007. J Stroke Cerebrovasc Dis 2013; 22:36-41.

Correspondence: Kameshwar Prasad

Department of Neurology Neurosciences Centre, All India Institute of Medical Sciences, Ansari Nagar, New Delhi, India

Tel: +91-11-26593497, 26588979, Fax: +91-11-26588663, 26588979

E-mail:kp0704@gmail.com

Received: January 28, 2015

Revised: March 12, 2015

Accepted: March 12, 2015

The authors have no financial conflicts of interest. 